\title{
Continuous-Flow Synthesis of Orange Emitting Sn(II)-Doped CsBr Materials
}

\author{
Samrat Das Adhikari, * Sofia Masi, Carlos Echeverría-Arrondo, Sara Miralles-Comins, \\ Rafael S. Sánchez, Jesum Alves Fernandes, Vladimir Chirvony, Juan P. Martínez-Pastor, \\ Victor Sans, * and Iván Mora-Seró**
}

\begin{abstract}
An ongoing demand toward lead-free all-inorganic cesium metal halide perovskites has presented $\mathrm{Sn}(\mathrm{II})$ as an ideal substitute of $\mathrm{Pb}$ (II) for applications in optoelectronic devices. The major concern regarding $\mathrm{Sn}(\mathrm{II})$ is the instability due to the ambient oxidation to $\mathrm{Sn}(\mathrm{IV})$. To expand the scope of traditional perovskite and analogues, herein the synthesis and optical performance of $\mathrm{Sn}$ (II)-doped CsBr, a new material formed by interstitial doping of Sn(II) into the $\mathrm{CsBr}$ matrix, are reported for the first time. This material is prepared following an antisolvent mediated recrystallization method using a continuous flow reactor, which is beneficial for scaling up the production compared to traditional batch reactors. $\mathrm{Sn}$ (II)-doped $\mathrm{CsBr}$ exhibits broadband orange emission with full-width-half-maximum of $180 \mathrm{~nm}$ and a photoluminescence quantum yield of $21.5 \%$. The emission turned to be highly stable over 7 months despite containing Sn(II). It is suggested that this is due to interstitial location of Sn(II) atoms in bulk of microcrystals. A broadband emission and high aerobic stability are attractive properties of the material for white-light emitting applications.
\end{abstract}

\section{Introduction}

In the past decade, lead halide perovskites are on the edge-ofthe-seat for their exciting power conversion efficiencies and

S. Das Adhikari, S. Masi, C. Echeverría-Arrondo, S. Miralles-Comins, R. S. Sánchez, V. Sans, I. Mora-Seró

Institute of Advanced Materials (INAM)

Universitat Jaume I. Av. de Vicent Sos Baynat

s/n 12071, Castelló de la Plana, Spain

E-mail:dasadhik@uji.es; sans@uji.es; sero@uji.es

J. A. Fernandes

School of Chemistry

University of Nottingham

Nottingham NG7 2RD, UK

V. Chirvony, J. P. Martínez-Pastor

Instituto de Ciencia de Materiales (ICMUV)

Universitat de Valencia

Paterna 46980, Spain

The ORCID identification number(s) for the author(s) of this article can be found under https://doi.org/10.1002/adom.202101024.

(C) 2021 The Authors. Advanced Optical Materials published by Wiley$\mathrm{VCH} \mathrm{GmbH}$. This is an open access article under the terms of the Creative Commons Attribution-NonCommercial-NoDerivs License, which permits use and distribution in any medium, provided the original work is properly cited, the use is non-commercial and no modifications or adaptations are made.

DOI: 10.1002/adom.202101024 display technologies, ${ }^{[1-6]}$ but their commercialization still faces significant challenges, as the toxicity of carcinogenic lead metal. ${ }^{[7,8]}$ As a suitable alternative, ongoing research is carrying out on leadfree metals such as $\mathrm{Sn}, \mathrm{Bi}, \mathrm{Ag}, \mathrm{Cu}$, In, and Sb-based perovskites and double perovskites, which also has proven their exceptional optoelectronic properties and promising solar to power energy conversion efficiencies. ${ }^{[9-18]}$ Among the different halide perovskites the fully inorganic are receiving an increasing attention due to their higher stability. ${ }^{19,20]}$ All-inorganic cesium tin halides present a variety of compositions, viz. $\mathrm{CsSnX}_{3}, \mathrm{Cs}_{4} \mathrm{SnX}_{6}$, $\mathrm{Cs}_{2} \mathrm{SnX}_{6}$ have been explored with good optoelectronic properties and photovoltaics. ${ }^{[21-23]}$ Importantly, $\mathrm{Sn}(\mathrm{II})$ is isovalent to $\mathrm{Pb}(\mathrm{II})$ and $\mathrm{CsSnX}_{3} / \mathrm{Cs}_{4} \mathrm{SnX}_{6}$ is isostructural with $\mathrm{CsPbX}_{3} / \mathrm{Cs}_{4} \mathrm{PbX}_{6}$. Hence tin can be considered as a satisfactory replacement to explore lead-free metal halide perovskites and analogues. ${ }^{[24]}$ Unfortunately, there is a limitation in tin-based perovskites owing to the oxidation of $\mathrm{Sn}^{2+}$ to $\mathrm{Sn}^{4+}$ upon exposure to ambient air and moisture, which degrades the material by creating trap states that irreversibly collapse the optical emission (and/or carrier lifetimes) of the material. This phenomenon somehow limits the commercial applicability of tin-based perovskites. To overcome this issue, the replacement of $\mathrm{Sn}^{2+}$ by $\mathrm{Sn}^{4+}$ was proposed as an alternative to get $\mathrm{Cs}_{2} \mathrm{SnX}_{6}$ with high valency of tin $\left(\mathrm{Sn}^{4+}\right)$. This typical structure of perovskites is referred to as vacancy-ordered double perovskites. ${ }^{[25]}$

In light of the emissive nature of the corresponding tin halide perovskites, $\mathrm{CsSnX}_{3}$ exhibits an excitonic emission with narrow full-width-half-maximum (FWHM). Unfortunately, they possess a very low photoluminescence quantum yield (PLQY) in comparison to the lead halide perovskites. ${ }^{[26]}$ However, current sign of progress on $0 \mathrm{D}$ tin halide perovskites have jumped up to importance in the field of optoelectronics due to their satisfactory photoluminescence with broad coverage across the visible region. ${ }^{[23]}$ Recently, several reports on $0 \mathrm{D} \mathrm{Cs}_{4} \mathrm{SnBr}_{6}$ published portraying a self-trapped excitonic (STE) emission originated from the isolated $\left[\mathrm{SnBr}_{6}\right]^{4-}$ octahedra. ${ }^{[27-31]}$ Typical characteristics of STE emissions are i) broad emission and ii) large stokes shift. ${ }^{[32]}$ Hence, the generation of white light due to covering a broad optical window and the reabsorption-related 
optical losses are ruled out here. However, these materials have some limitations in terms of broad application and commercialization because of their ambient instability. These materials transform to $\mathrm{Cs}_{2} \mathrm{SnBr}_{6}$ in presence of air, subsequently lose the emission. ${ }^{[28]} \mathrm{Cs}_{2} \mathrm{SnX}_{6}$, vacancy ordered double perovskites are a class of materials, where the $\mathrm{Sn}$ atom has a +4-oxidation state and hence they possess higher stability in the open air. ${ }^{[18]}$ Their optical performances enable many application segments including photovoltaics and light-emitting materials. Unfortunately, the potential optoelectronic application is also break-off here due to experiencing very low PLQY. ${ }^{[18,33]}$

For the preparation of cesium tin halide perovskite and analogues, antisolvent mediated recrystallization is the easiest method followed previously. ${ }^{[29]}$ Nevertheless, the labor intensive batch methodology is cumbersome, time consuming and presents limited scalability. Hence, we choose flow-reactors to have an accurate control over the crystallization conditions by finely tuning the flow rates of the precursors and antisolvent solutions. Flow-reactors have been used to synthesize and to crystallize nanomaterials and $\mathrm{APbX}_{3}(\mathrm{~A}=$ formadinium/methylammonium/Cs) perovskite nanocrystals and it was established that these methods are favorable for higher reproducibility and scalable production. ${ }^{[34-40]}$ Continuous flow system can be modulated controlling flow rates, temperatures and pressures to mix a homogeneous precursor solution/organic ligand(s)/antisolvent in micromixers and microreactors, with the possibility to finely tune residence times. Herein, we have prepared $\mathrm{Cs}_{4} \mathrm{SnBr}_{6}$ and $\mathrm{Sn}(\mathrm{II})$-doped $\mathrm{CsBr}$ following a simple flow-reactor by setting down commands of different precursor and antisolvent flow rates. The former material is well-studied in accordance to their STE emission, A-site doping, and thermographic applications. ${ }^{[28-31,41]}$ We found $\mathrm{Cs}_{4} \mathrm{SnBr}_{6}$ is unstable and it degrades in open air over time and eventually transforms to $\mathrm{Cs}_{2} \mathrm{SnBr}_{6}$. However, the other material, $\mathrm{Sn}(\mathrm{II})$-doped
$\mathrm{CsBr}$ was prepared for the first time, which exhibits broadrange $\mathrm{STE}$ emission (PLQY $=21.5 \%, \mathrm{FWHM} \approx 180 \mathrm{~nm}$ ) and highly stable in the open air over 7 months as per laboratory report.

\section{Result and Discussions}

For the material synthesis the room-temperature antisolvent mediated recrystallization method was followed, where dimethyl formaldehyde (DMF) was used to dissolve the precursors and toluene to precipitate the crystalline material. The fundamental of this process includes dissolving precursor solutes into a polar (or good) solvent and by the incorporation of an antisolvent (or poor) dissolved ions arrange among themselves to form an energetically favorable crystalline material. We modified the synthetic procedure by changing from batch to continuous flow. Two high performance liquid chromatography pumps were used to flow the precursors dissolved in DMF and the toluene to induce the crystallization. These two pumps were connected with 1/16" T-mixer (Swagelok), followed by a short tubular microreactor, which served to rapidly mix the precursor solution and the antisolvent, followed by the collection of the crystalline powder at the outlet. The schematic of the flow-reactor is displayed on Figure 1a. The flow rate of the

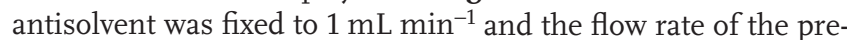
cursor solution was changed from 0.1 to $0.5 \mathrm{~mL} \mathrm{~min}^{-1}$. Interestingly, we obtained two different products at different precursor flow rates (depicted in Figure S1, Supporting Information). We observed the preferential formation of one product at the lower flow rates of precursors solution $\left(0.1-0.2 \mathrm{~mL} \mathrm{~min}^{-1}\right)$ and another at higher flow rates $\left(0.3-0.5 \mathrm{~mL} \mathrm{~min}{ }^{-1}\right)$, respectively. At lower flow rates, $\mathrm{Cs}_{4} \mathrm{SnBr}_{6}$, a white-colored air-unstable greenemitting material was formed, whose emission was quenched (a)

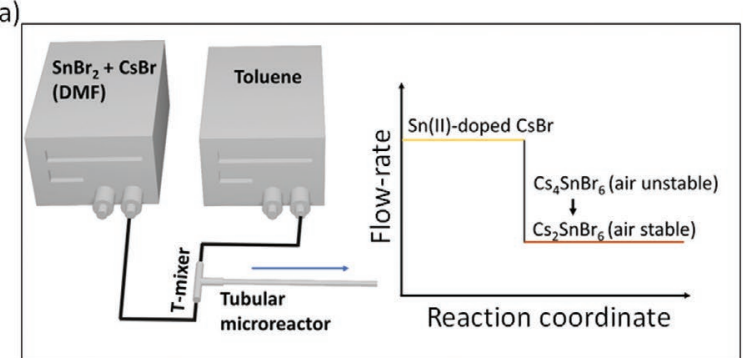

(c)

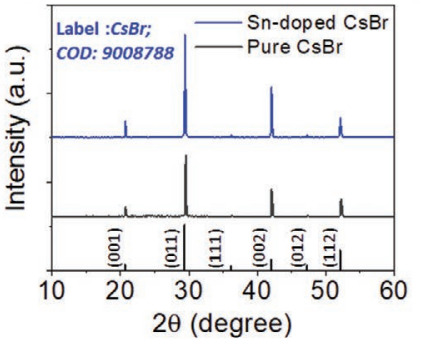

(d)

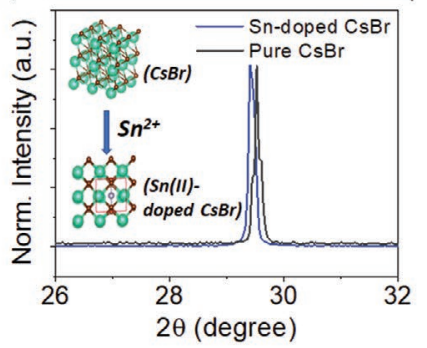

(b)

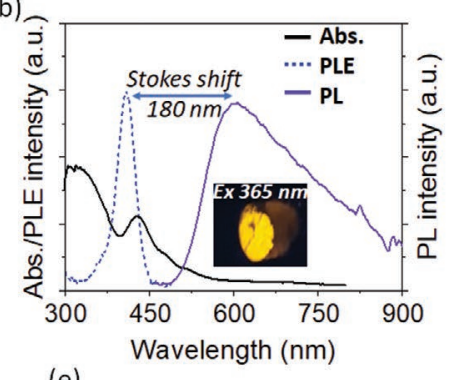

(e)

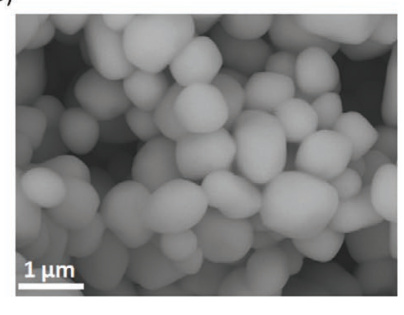

Figure 1. a) Schematic presentation of experimental setup and typical reaction procedure. b) UV-vis absorbance (Abs.), PL, and PLE spectra of Sn(II)doped CsBr powder. Inset shows illuminated orange emission of Sn(II)-doped CsBr under 365 nm UV-lamp. c) XRD patterns of Sn(II)-doped CsBr and undoped CsBr. The label of XRD for matching is referenced from COD: 9008788. d) Enlarged view of (011) peak to show the peak shifting belongs to lattice expansion for $\mathrm{Sn}(\mathrm{II})$-dopant incorporation into the $\mathrm{CsBr}$ lattice. e) SEM image of as-prepared Sn(II)-doped CsBr powder. 
during the drying process and transformed to $\mathrm{CsBr}$ and $\mathrm{Cs}_{2} \mathrm{SnBr}_{6}$ in the open air. The white-colored $\mathrm{Cs}_{4} \mathrm{SnBr}_{6}$ turned to orange-colored due to the formation of $\mathrm{Cs}_{2} \mathrm{SnBr}_{6}$ in the open air. At higher flow rates, $\mathrm{Sn}(\mathrm{II})$-doped $\mathrm{CsBr}$, a yellowishcolored powder was precipitated, which possessed a strong orange emission found to be stable over 7 months. The digital images of $\mathrm{Sn}(\mathrm{II})$-doped $\mathrm{CsBr}$ powder and the oxidized powder $\left(\mathrm{Cs}_{4} \mathrm{SnBr}_{6} \mathrm{Cs}_{2} \mathrm{SnBr}_{6}\right)$ are shown in Figure S2a,b in the Supporting Information. The illuminated images of orange emitting $\mathrm{Sn}$ (II)-doped $\mathrm{CsBr}, \mathrm{Cs}_{4} \mathrm{SnBr}_{6}$ (under inert atmosphere) and their poly(methyl methacrylate) (PMMA)-coated films on top of glass slides under the $365 \mathrm{~nm}$ UV-lamp are provided in Figure $\mathrm{S} 3 \mathrm{a}-\mathrm{C}$ in the Supporting Information.

Figure 1b presents the optical absorbance (Abs.), photoluminescence (PL), and PL excitation spectrum (PLE) of as-obtained $\mathrm{Sn}(\mathrm{II})$-doped $\mathrm{CsBr}$. The figure inset shows the digital image of the orange light emission from $\mathrm{Sn}(\mathrm{II})$-doped $\mathrm{CsBr}$ powders under a $365 \mathrm{~nm}$ excitation. For PL measurements, the material was excited at $420 \mathrm{~nm}$, and for PLE, the detection wavelength was fixed at $600 \mathrm{~nm}$. This emission exhibits large FWHM $(\approx 180 \mathrm{~nm})$ and Stokes shift $(180 \mathrm{~nm})$.

The X-ray diffraction (XRD) pattern of $\mathrm{Sn}(\mathrm{II})$-doped $\mathrm{CsBr}$ synthesized at high flow rate $\left(0.4 \mathrm{~mL} \mathrm{~min}^{-1}\right)$ exactly matched with cubic $\mathrm{CsBr}$ (Fm-3m space group). A control reaction was carried out without employing $\mathrm{SnBr}_{2}$ into the reaction and as-obtained undoped material was characterized as the same XRD pattern (see Figure 1c). The crystalline phase is matched with CsBr COD:9008788. A XRD peak shifting toward the lower angle was observed indicating a lattice expansion (see Figure 1d). From the theoretical calculations (explained in a later section), it is shown that doping $\mathrm{Sn}^{2+}$ is incorporated at the interstitial position of the $\mathrm{CsBr}$ lattice, in good agreement with the lattice expansion observed in Figure 1d. The morphology of the as-obtained $\mathrm{Sn}(\mathrm{II})$-doped $\mathrm{CsBr}$ material was monitored by scanning electron microscope (SEM) and transmission electron microscopic (TEM) imaging (Figure 1e and Figure S4, Supporting Information), which shows quasi spherical particles (300 $\left.\mathrm{nm}^{-1} \mu \mathrm{m}\right)$.

We have systematically studied the formation of materials under different precursor solution flow rates and we analyzed the results by UV-vis absorption spectroscopy (Figure 2a). The flow rate ratio is defined as $\mathrm{X} / \mathrm{Y}$ where $\mathrm{X}$ and $\mathrm{Y}$ are the precursor $\left(\mathrm{SnBr}_{2}+\mathrm{CsBr}\right)$ and the antisolvent (toluene) flow rates, respectively. At lower flow rate ratios $(0.1 / 1$ and $0.2 / 1)$, there is an absorbance peak near $340 \mathrm{~nm}$, which belongs to $0 \mathrm{D}$ $\mathrm{Cs}_{4} \mathrm{SnBr}_{6}$ perovskite, referenced from the literature report. ${ }^{[27]}$ For the sample prepared at $0.2 / 1$ ratio, an evolution of the broad absorbance peak near $430 \mathrm{~nm}$ is observed, which is due to the formation of $\mathrm{Sn}(\mathrm{II})$-doped $\mathrm{CsBr}$, see Figure 2a. The $430 \mathrm{~nm}$ peak intensifies and the $340 \mathrm{~nm}$ peak disappears finally with increasing flow rate ratio that confirms the formation of only $\mathrm{Sn}$-doped $\mathrm{CsBr}$ product at high flow rate ratios (green and blue lines line Figure 2a for 0.3/1-0.4/1 and Figure S5a, Supporting Information, for $0.5 / 1)$. Corresponding PL and PLE spectra of these sets of materials are presented in Figure 2b. The materials were excited at $420 \mathrm{~nm}$ to detect PL. The materials
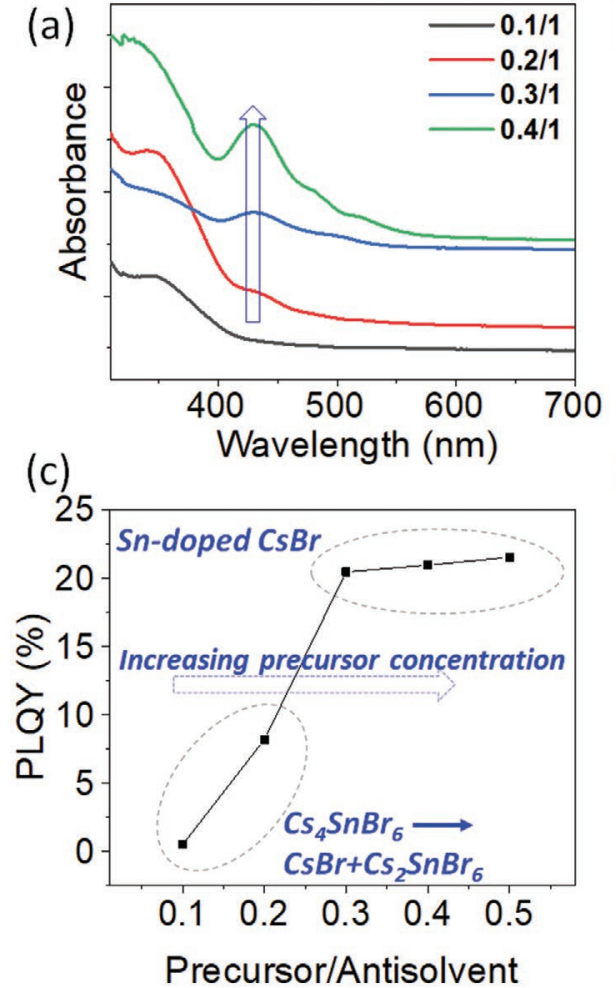

(b)

(d)
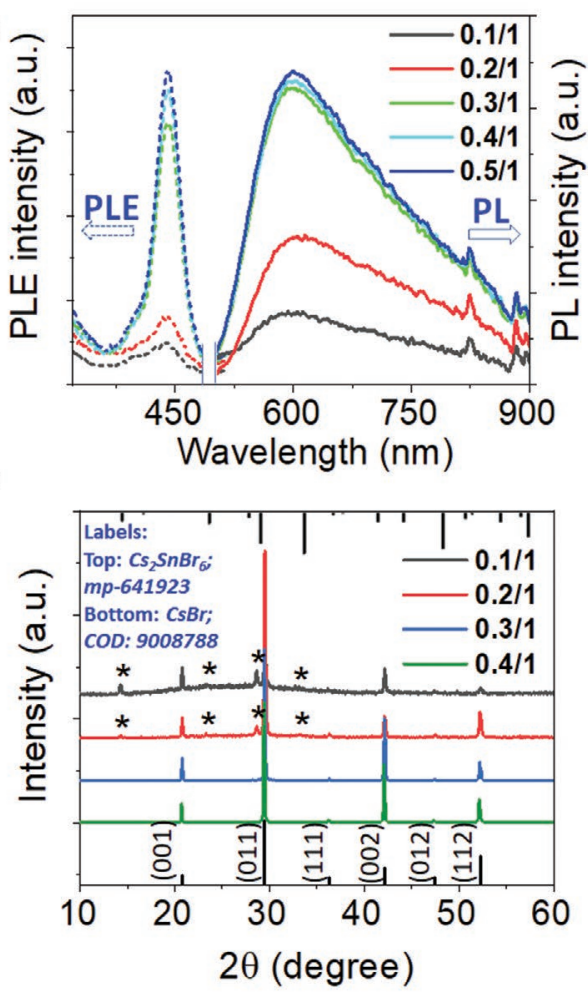

Figure 2. a) UV-vis absorbance and b) PL (solid lines) and PLE (dashed lines) of samples prepared at different flow rates. PL spectra were obtained under the excitation of $420 \mathrm{~nm}$. c) A plot of PLQY versus precursor/antisolvent flow ratio. Samples were excited at $420 \mathrm{~nm}$ for the PLQY measurements. d) XRD patterns of powder samples prepared at different flow rates. Peaks belonging to $\mathrm{Cs}_{2} \mathrm{SnBr}_{6}$ are marked with asterisk. 
prepared at low flow rate ratios exhibit a low intensity PL, that increases at higher flow rate ratios, stabilizing the intensity for flow rate ratios higher than $0.3 / 1$. The orange-emitting material shows a significantly narrow PL excitation range between 390 and $450 \mathrm{~nm}$, beyond which the material is barely luminescent or even nonluminescent at all, which is unusual for band-to-band emitting semiconductors. The PLQYs of these samples were measured and plotted versus the flow rate ratio that is presented in Figure 2c, where a highest PLQY of 21.5\% was observed at higher flow rate ratios. All the obtained PLQY values are provided in Table S1 in the Supporting Information. The excitation wavelength was $420 \mathrm{~nm}$ for the PLQY measurements. The poor orange PL obtained at lower flow rate ratios is due to the preferential crystallization of $\mathrm{Cs}_{4} \mathrm{SnBr}_{6}$, which has a green emission under the excitation of $337 \mathrm{~nm}$, see Figure S6 in the Supporting Information, and a minimum formation of Sn(II)-doped CsBr. Table S2 in the Supporting Information provides the corresponding PLQY of the green emission from $\mathrm{Cs}_{4} \mathrm{SnBr}_{6}$ (excitation wavelength $337 \mathrm{~nm}$ ). Contrarily, it was found that the intensity of the green emission decreased with increasing precursor flow rate ratio (Figure S6a, Supporting Information).

The as-prepared samples were dried in the ambient conditions for the XRD characterization, presented in Figure 2d. The XRD patterns of all of these samples were matched with $\mathrm{CsBr}$ (Pm-3m space group, cubic phase). At lower flow rates, additional peaks were observed (marked with asterisk in Figure 2d, first two patterns from the top), which belonged to the $\mathrm{Cs}_{2} \mathrm{SnBr}_{6}$ compound (mp-641923, triclinic phase). At higher flow rate ratios $(0.3 / 1-0.5 / 1)$, no additional peaks were observed for any of the samples corresponding to the phasepure $\mathrm{CsBr}$ cubic lattice without any impurities (Figure 2d, last two patterns at the bottom for $0.3 / 1-0.4 / 1$ and Figure S5b, Supporting Information, for $0.5 / 1$ ). In an alternative way, the unstable green-emitting product obtained at $0.1 / 1$ was collected under the $\mathrm{N}_{2}$ and it was coated with PMMA to prevent it from ambient atmosphere. Figure S7 in the Supporting Information presents the XRD pattern of the PMMA-coated green emitting product, which matches with the $\mathrm{Cs}_{4} \mathrm{SnBr}_{6}$ crystal phase. The ambient instability of the green-emitting $\mathrm{Cs}_{4} \mathrm{SnBr}_{6}$ is due to the oxidation of $\mathrm{Sn}(\mathrm{II})$ to $\mathrm{Sn}(\mathrm{IV})$ and it transformed to $\mathrm{Cs}_{2} \mathrm{SnBr}_{6}$ and $\mathrm{CsBr}^{\left[{ }^{[28]}\right.}$ The powder samples prepared at low flow rates only have the XRD patterns for $\mathrm{Cs}_{2} \mathrm{SnBr}_{6}$ (do not confuse with PMMA-coated sample). Beyond 0.3/1, the XRD patterns only belong to $\mathrm{CsBr}$, confirms the formation of phase-pure $\mathrm{Sn}$ (II)doped $\mathrm{CsBr}$. Indeed, it was discarded the possibility of the new material being $\mathrm{Cs}_{2} \mathrm{SnBr}_{6}$, the oxidized product of $\mathrm{Cs}_{4} \mathrm{SnBr}_{6}$, by separately synthesizing $\mathrm{Cs}_{2} \mathrm{SnBr}_{6}$ by wet-chemical method (see the Experimental Section), and comparing the XRD patterns (Figure S8, Supporting Information). This confirmed that $\mathrm{Cs}_{2} \mathrm{SnBr}_{6}$ phase shows cubic phase and $\mathrm{Fm}-3 m$ space group (mp-641923) as a difference from the new material. This information explained the phase of orange emitting material is identical to $\mathrm{CsBr}$ and not belongs to $\mathrm{Cs}_{2} \mathrm{SnBr}_{6}$.

Raman spectroscopic measurements were carried out to study the $\mathrm{Sn}-\mathrm{Br}$ vibration into the crystal lattice. Herein, comparing the as-obtained spectra of the dried powder samples obtained at 0.1/1 and 0.5/1 are presented in Figures 3a,b, respectively. Surprisingly, we observed a discrepancy in the Raman spectrum pattern depending on the flow rate ratios. (a)

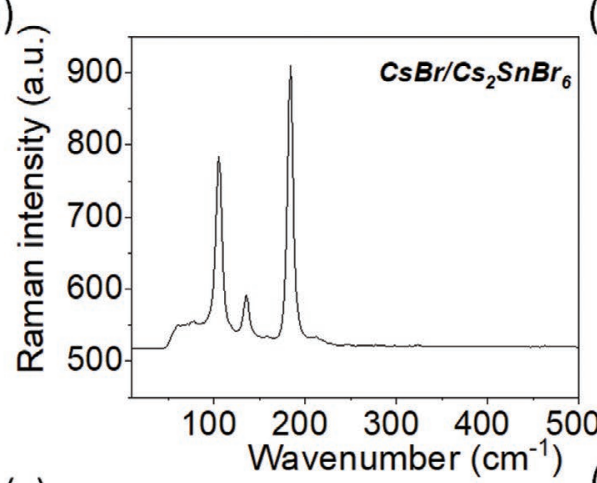

(c)

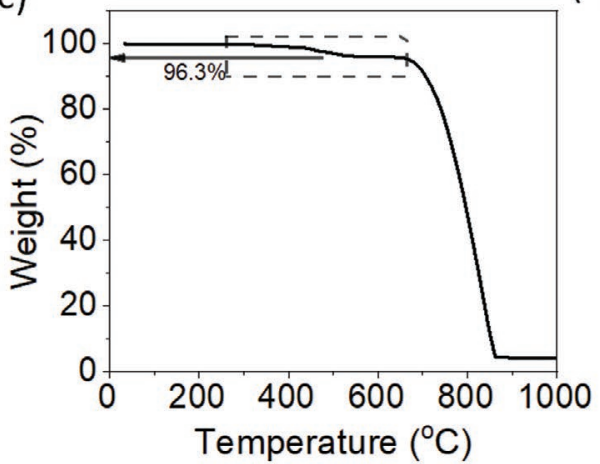

(b)
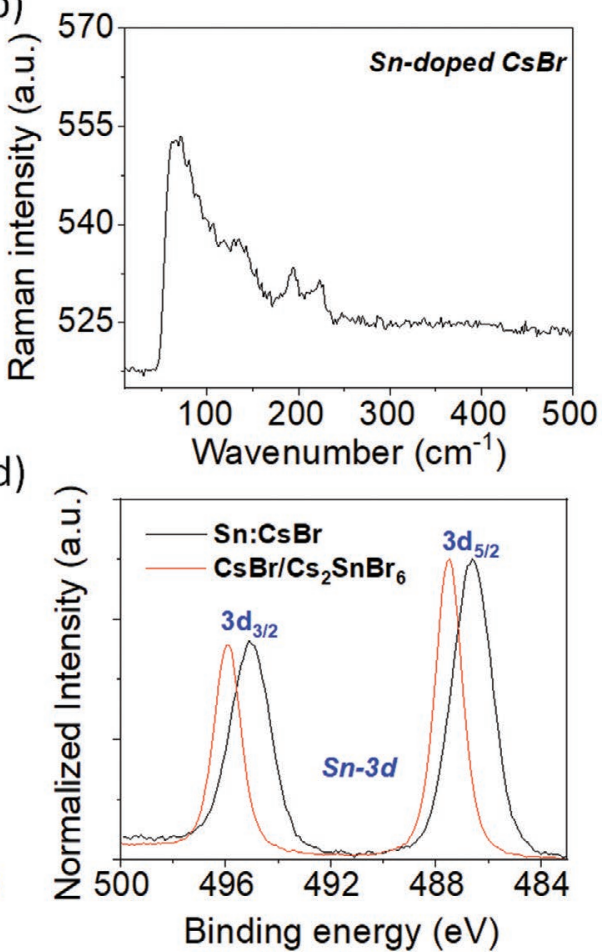

Figure 3. Raman spectra of a) $\mathrm{CsBr}_{+} \mathrm{Cs}_{2} \mathrm{SnBr}_{6}$ (oxidized from $\mathrm{Cs}_{4} \mathrm{SnBr}_{6}$ ) and b) $\mathrm{Sn}$ (II)-doped $\mathrm{CsBr}$ powder samples. c) $\mathrm{TGA}$ of $\mathrm{Sn}(\mathrm{II})$-doped $\mathrm{CsBr}$ powder. d) XPS of Sn-3d of $\mathrm{Sn}(\mathrm{II})$-doped $\mathrm{CsBr}$ (black) and $\mathrm{CsBr}+\mathrm{Cs}_{2} \mathrm{SnBr}_{6}$ (red; oxidized from $\mathrm{Cs}_{4} \mathrm{SnBr}_{6}$ ). 
At $0.1 / 1$, there are three intense peaks at 106.9, 136.1, and $184.3 \mathrm{~cm}^{-1}$ while those are absent for the sample prepared at $0.5 / 1$. As discussed earlier, due to ambient oxidation, greenemitting $\mathrm{Cs}_{4} \mathrm{SnBr}_{6}$ transformed to $\mathrm{CsBr}$ and $\mathrm{Cs}_{2} \mathrm{SnBr}_{6}$. In $\mathrm{Cs}_{2} \mathrm{SnBr}_{6}$, there are three Raman active vibrational modes that are present due to the vibrations inside the $\left[\mathrm{SnBr}_{6}\right]^{2-}$ octahedra, which are the $\mathrm{Br}-\mathrm{Sn}-\mathrm{Br}$ asymmetric bending, $\mathrm{Sn}-\mathrm{Br}$ asymmetric stretching, and symmetric $\mathrm{Sn}-\mathrm{Br}$ stretching vibration. ${ }^{[42]}$ In the case of Sn-doped $\mathrm{CsBr}, \mathrm{Sn}(\mathrm{II})$ is occupying interstitial sites, as we discuss below, in the CsBr crystal lattice and conjugated to the Br-atoms linearly (Figure S9, Supporting Information). Hence, such vibrational modes are absent for the interstitially $\mathrm{SnBr}_{2}$ (doped) units into the $\mathrm{CsBr}$ lattice.

Thermogravimetric analysis (TGA) presented in Figure 3c also predicts a similar result for the presence of a minimum content of $\mathrm{Sn}$, as $\mathrm{Sn}$-doped $\mathrm{CsBr}$ has a 3.7\% mass loss near $530{ }^{\circ} \mathrm{C}$ and rest is decomposed at $780^{\circ} \mathrm{C}$. A control experiment of undoped $\mathrm{CsBr}$ confirms the rest of the mass lost appertain to $\mathrm{CsBr}$ (see Figure S10, Supporting Information). The initial loss for the dopant ion inclusion now led us to calculate the dopant ion percentage into the as-prepared material and hence, we have calculated $10 \%$ of initial mass loss for the material synthesized at $0.1 / 1$, which indeed $\mathrm{Cs}_{4} \mathrm{SnBr}_{6}$ transformed to $\mathrm{CsBr}$ and $\mathrm{Cs}_{2} \mathrm{SnBr}_{6}$ (see Figure S11, Supporting Information). Hence, from the synthesis point of view, a high amount of $\mathrm{SnBr}_{2}$ was precipitated at low precursor flow rates, whereas the tin content decreased in the final material with the increase of precursor concentration (simultaneously, the DMF content). Elemental composition was further verified using energy dispersive X-ray spectroscopy (EDS) analysis from the TEM measurements, which estimates the Sn-percentage from 1.3-3.8\% (Figure S12 and Table S3, Supporting Information).

X-ray photoelectron spectroscopy (XPS) was carried out to study the oxidation state of Sn-dopants into the CsBr crystal. Figure 3d presents the Sn-3d XPS peaks of Sn-doped CsBr (black) and $\mathrm{CsBr}+\mathrm{Cs}_{2} \mathrm{SnBr}_{6}$ (red) oxidized from the initial formation of $\mathrm{Cs}_{4} \mathrm{SnBr}_{6}$. Binding energy of $\mathrm{Sn}-3 \mathrm{~d}_{5 / 2}$ for $\mathrm{Sn}$-doped $\mathrm{CsBr}$ appears at 486.6 ascribed to $\mathrm{Sn}^{2+}$ in $\mathrm{Sn}$-doped $\mathrm{CsBr}$ whereas $\mathrm{CsBr}+\mathrm{Cs}_{2} \mathrm{SnBr}_{6}$ appears at higher binding energy $487.5 \mathrm{eV}$ associated to $\mathrm{Sn}^{4+}$, which was expected due to the oxidized powder. ${ }^{[43]}$

Eventually, in order to confirm the exact nature of the material prepared at high flow ratios, the system was further investigated by density functional theory (DFT) calculations performed with Quantum ESPRESSO, ${ }^{[4]}$ a plane-wave periodic DFT code. First, we geometrically relaxed a clean unit cell composed of 54 $\mathrm{CsBr}$ atoms using a single $k$ point ( $\Gamma$ for the direct bandgap), spin-orbit interactions, and PBEsol pseudopotentials generated with the generalized gradient approximation (GGA) for the exchange-correlation functional. The $\mathrm{Cs}-\mathrm{Br}$ bond length in the geometrically relaxed atomic structure is $3.80 \AA$, $2.2 \%$ larger than the experimental $3.72 \AA$. Secondly, we doped the unit cell with an interstitial $\mathrm{Sn}$ atom placed between two $\mathrm{Br}$ atoms at the central region of a quasi-orthorhombic void space, see Figure 4a, and relaxed the atomic structure again, resulting in a $\mathrm{Sn}-\mathrm{Br}$ distance of $2.94 \mathrm{~A}$. The considered 3.7\% doping ratio was previously estimated in an experiment consisting in heating synthesized powder samples of Sn-doped $\mathrm{CsBr}$ and measuring the weight loss (see Figure 3c). The calculated densities of states (DOS) for this system, addressed in Figure 4a, reveal a bandgap of $4 \mathrm{eV}$ which underestimates the experimental $7.3 \mathrm{eV}^{[45]}$ as usual when DFT computations are run at the GGA level, however allows to observe the trends caused by $\mathrm{Sn}$ addition. The DOS show as well two peaks of p-like character within the bandgap, appended by the dopant: the left-hand one is formed by two polarons at two degenerate energy levels, indicating a +2 oxidation state for the Sn atom, and the right-hand one is empty and also doubly degenerate. The unoccupied peak is placed at $2.7 \mathrm{eV}$ above the valence band minimum (VBM); this energy value is close and consistent with the $2.9 \mathrm{eV}(430 \mathrm{~nm})$ peak obtained in the experimental absorption spectrum. Furthermore, the occupied lowest-energy states show a characteristic ring shape (quantum numbers $l=+1$ and $m=-1$ ), and the empty states in the second peak are also ring shaped $(l=+1$ and $m=+1)$. Since impurity states are strongly localized at the Sn site, the photoexcitation of one electron from the VBM into these states would result in self-trapped excitons, that is excitons hooked to the crystal lattice. Moreover, by looking at the DOS of Figure 4a and the local contributions of the Sn dopant and the two $\mathrm{Br}$ neighboring atoms, we conclude that the Sn atomic orbitals are only slightly hybridized with the host crystal states, in consonance with the interstitial nature of the dopant.

We have further obtained theoretical XRD patterns for the clean and doped cases. The deviation of the main peak in the XRD pattern of the doped structure toward the left relative to the same peak in the spectrum of the clean host crystal is $0.4^{\circ}$. This value is larger than the $0.12^{\circ}$ shift obtained in the XRD experiments (Figure 1d), what would indicate that the real concentration of Sn dopants in the synthesized powder samples is smaller than the initially estimated 3.7\%.

PL spectra of Sn(II)-doped CsBr measured in 200-330 K are shown in Figure 5a. Temperature increase in the indicated diapazon results in approximately twofold decrease of PL integral intensity, which is accompanied by similar twofold decrease of the main (shorter) component lifetime for kinetics measured at 600 and $700 \mathrm{~nm}$ (Figures S13 and S14, Supporting Information). The found temperature dependence can be explained by freezing nonradiative deactivation channels at lower temperatures, which are activated with temperature. Another interesting aspect regarding stability is presented in Figure $5 \mathrm{~b}$. The material exhibits long-term optical stability and hence an identical emission intensity even after the 7 months. This material belongs to the $\mathrm{Sn}$ (II) family and hence it has extended longterm stability and possibly in competition with lead halide perovskites. Presumably, the extended stability is due to i) doping at the interstitial position not facing the surface and ii) protective covering by the $\mathrm{Cs}-\mathrm{Br}$ crystal matrix.

To further elucidate this newly explored orange emitting material for light-emitting diode (LED) applications, herein, we dip-coated Sn-doped $\mathrm{CsBr}$ on top of a commercially available $430 \mathrm{~nm}$ blue chip. The electroluminescence spectra were detected at different voltage and presented in Figure $5 \mathrm{c}$. The blue electroluminescence at $430 \mathrm{~nm}$ from the commercial chip further excites $\mathrm{Sn}$-doped $\mathrm{CsBr}$ and gives rise to its characteristic emission identical to the PL. In the inset, a zoom view of the emission region belonging to the $\mathrm{Sn}$-doped $\mathrm{CsBr}$ is shown. A Commission Internationale de l'Éclairage color coordinate is provided in Figure S15 in the Supporting Information to show the contribution of orange emission. The 

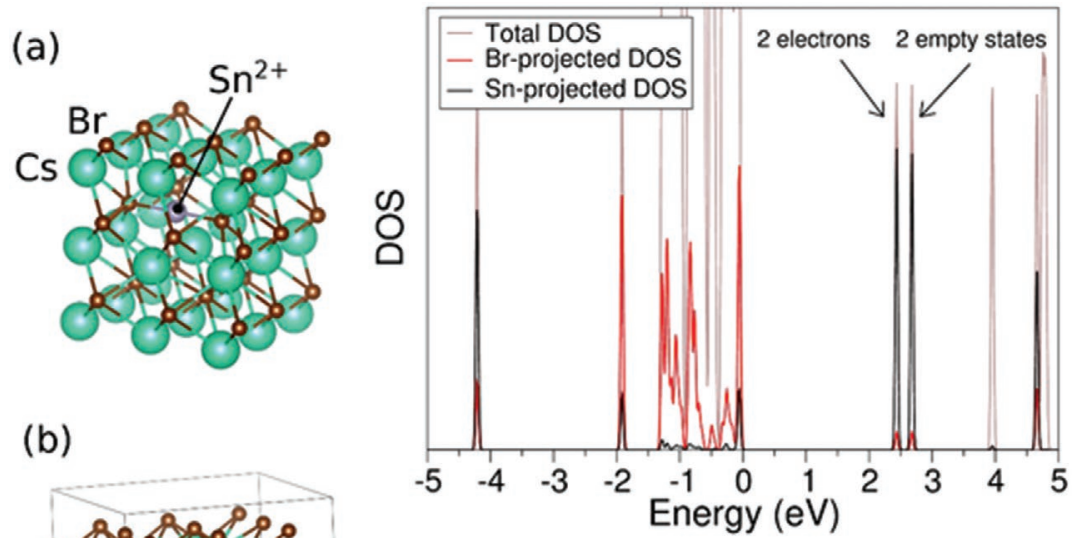

(b)
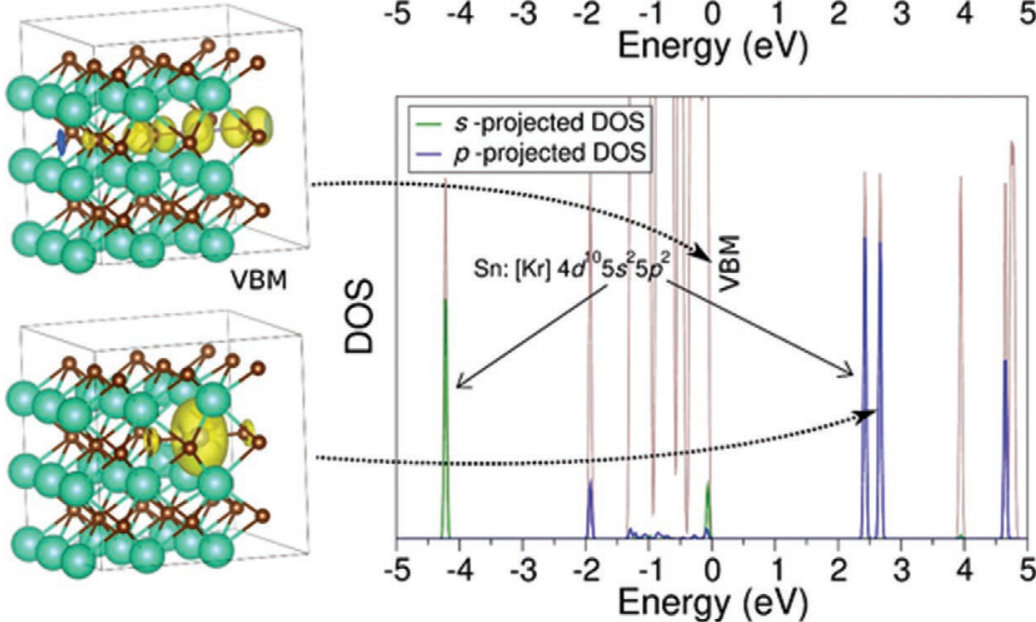

Figure 4. a) Supercell of cubic $\mathrm{CsBr}$ with an interstitial $\mathrm{Sn}$ dopant centered in an orthorhombic void with two $\mathrm{Br}$ atoms aside. Total densities of states (DOS) with indication of the Sn contribution. b) Total DOS with indication of the sp-contributions of the dopant. Isosurfaces of constant charge density for a self-trapped exciton composed of a hole at the valence band maximum (VBM, upper panel) and the promoted electron occupying one of the available impurity states (lower panel).

broad spectral characteristics of the PL signals and the moderate to good PLQY values obtained from the Sn-doped $\mathrm{CsBr}$ material, together with the outstanding (photo-)chemical stability suggests that this material could be a good candidate to be exploited as a down-converter toward the developing of white light-emitting devices. Additionally, our strategy opens up a new paradigm for developing a new family of materials, through a systematic compositional screening, suitable for optoelectronic applications.
In order to qualitatively quantify the scope of the continuous flow reaction methodology in particular study, a parallel reaction was carried out manually by the dropwise addition of $0.5 \mathrm{~mL}$ of precursor solution to $1 \mathrm{~mL}$ of antisolvent (toluene) under continuous stirring conditions. The product obtained by this manual procedure is also characterized as $\mathrm{Sn}(\mathrm{II})$-doped $\mathrm{CsBr}$ from the XRD. In comparison to the flow reactor method, the material obtained from the manual reaction procedure has less optical performance, and poor particle shape definition
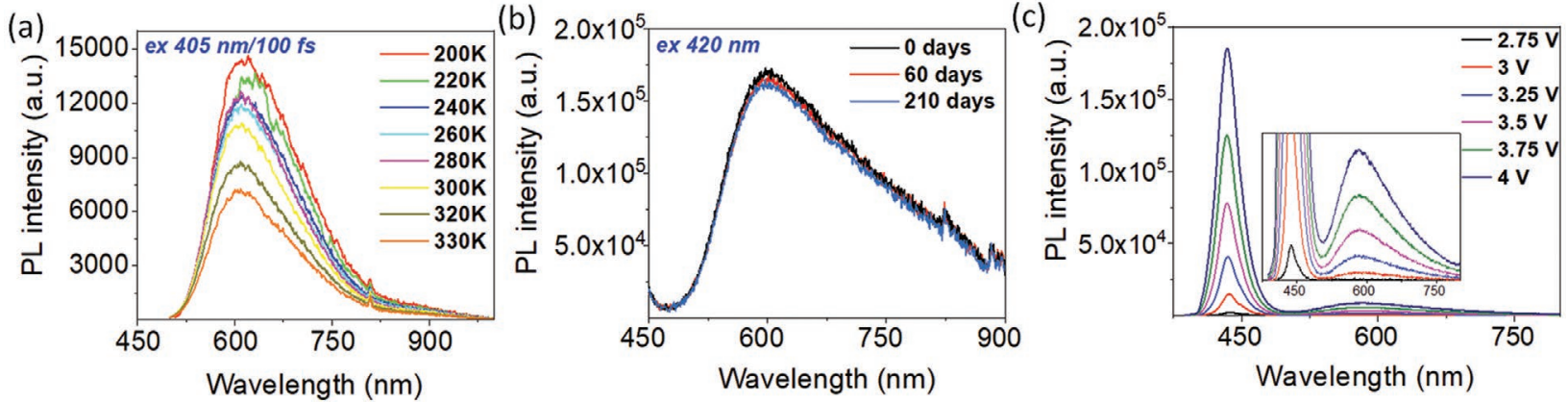

Figure 5. a) PL spectra of $\mathrm{Sn}$ (II)-doped $\mathrm{CsBr}$ measured under $405 \mathrm{~nm}$ excitation by $200 \mathrm{fs}$ laser pulses at different temperatures from 200 to $330 \mathrm{~K}$. b) PL spectra of Sn(II)-doped CsBr measured at different times after synthesis. c) Down-converted PL spectra of Sn(II)-doped CsBr excited by $430 \mathrm{~nm}$ electroluminescence of a commercial blue chip made of $(\mathrm{InGa}) \mathrm{N}$. 
and size distribution. A comparison to the optical emission and the SEM images is provided in Figures S16 and S17 in the Supporting Information. In Figure S17 in the Supporting Information, SEM image of undoped $\mathrm{CsBr}$ shows an identical shape and size distribution to the $\mathrm{Sn}(\mathrm{II})$-doped $\mathrm{CsBr}$ prepared using flow-reactor. This is due to the improved mixing achieved under continuous-flow conditions, which is particularly relevant for fast reactions. This procedure allows to generate advanced nanostructured materials under steady state. Hence, the flow reactor is much more effective toward i) production of mass-scale continuously, ii) formation of material at short-time scale leads to the formation of equal-shaped nanocrystals, and iii) enhanced optical performance of crystalline products.

\section{Conclusion}

In summary, we have prepared a new material belonging to the lead-free cesium metal halide family, which is prepared using a continuous flow reactor. By altering the precursor flow rates, which is indeed the ratio of precursor concentration to the antisolvent, we obtained a tin-rich phase $\mathrm{Cs}_{4} \mathrm{SnBr}_{6}$ at low precursor concentration. With the increase of precursor concentration by increasing the precursor flow rate, a lesser amount of tin was precipitated along with the $\mathrm{CsBr}$, which led to the formation of the new material, Sn(II)-doped CsBr. This material possesses a broadband STE emission at the orange-red region, which emits PL with a PLQY up to $21.5 \%$ if excited in the $390-435 \mathrm{~nm}$ diapason. This material has a visible-light absorbance near $2.8 \mathrm{eV}$ due to mid-gap localized states contributed by the dopant which were further confirmed by quantum mechanical calculations based on the density functional theory. It is worth to highlight, that $\mathrm{Sn}(\mathrm{II})$-doped $\mathrm{CsBr}$ is highly stable in the open air, and its emission was found stable over 7 months. The present stateof-art of this finding leads us to the proof of concept for the lead-free cesium metal halides as a light-emitting (down-shift wavelength converter) material. The broadband emission from this material could be implemented for white-LED applications.

\section{Experimental Section}

Preparation of Precursor Solution: $212 \mathrm{mg}(1 \mathrm{mmol})$ of $\mathrm{CsBr}$ and $278 \mathrm{mg}(1 \mathrm{mmol})$ of $\mathrm{SnBr}_{2}$ were added to $25 \mathrm{~mL}$ of DMF and stirred for $30 \mathrm{~min}$ to obtain a clear solution. Finally, this solution was used as the precursor solution.

Flow-Reactor Synthesis: Two different flow pumps were used and connected with a "T" junction. Herein, toluene was used as the antisolvent. Toluene was pumped using one of the pumps connected parallel to the product-side capillary, and precursor solution was pumped using another pump, which was connected perpendicular to the product-side capillary. The flow-rate of toluene was fixed to $1 \mathrm{~mL} \mathrm{~min} \mathrm{~m}^{-1}$ and the flow-rate of precursor solution was changed from 0.1 to $0.5 \mathrm{~mL} \mathrm{~min}$. Details of flowrate and obtained different products are summarized in Table 1.

Preparation of $\mathrm{Cs}_{2} \mathrm{SnBr}_{6}: 212 \mathrm{mg}$ of $\mathrm{CsBr}$ and $278 \mathrm{mg}$ of $\mathrm{SnBr}_{2}$ were added in $2 \mathrm{~mL}$ of acetone and stirred for overnight at $50{ }^{\circ} \mathrm{C}$. A brown colored powder was formed.

PMMA-Coating for XRD Characterization of As-Prepared $\mathrm{Cs}_{4} \mathrm{SnBr}_{6}$ : At $0.1 / 1 \mathrm{P} / \mathrm{A}$, the green emitting material was collected under $\mathrm{N}_{2}$ atmosphere after $10 \mathrm{~min}$ of flow (total volume $=11 \mathrm{~mL}$ ), finally the obtained material was kept inside the glove box in an air-tightened glass vial and settle the product + solution. After 45-60 min, the supernatant was decanted and the precipitate was collected. In another vial, $100 \mathrm{mg}$ of PMMA and $1 \mathrm{~mL}$ of chlorobenzene was loaded together, heated to $60{ }^{\circ} \mathrm{C}$ for $30 \mathrm{~min}$, poured to the collected $\mathrm{Cs}_{4} \mathrm{SnBr}_{6}$ product once a clear solution of PMMA obtained. It was stirred for another $30 \mathrm{~min}$. Finally, the product embedded in PMMA+chlorobenzene solution spin-coated on top of a glass slide, dried to ambient condition. Noteworthy, all these procedures were carried out inside the glove box. This film was also found to be stable over 7 months.

Characterizations: UV-vis absorption spectra were collected from Perkin Elmer UV-vis-NIR spectrophotometer, the powder samples were measured using an integrated sphere unit. PL and PLQY measurements were carried out using an integrating sphere unit (Hamamatsu). The samples were measured in a powder form. The Sn(II)-doped $\mathrm{CsBr}$ samples were excited at $420 \mathrm{~nm}$ and $\mathrm{Cs}_{4} \mathrm{SnBr}_{6}$ samples were excited at $340 \mathrm{~nm}$. PLE was measured using fluorimeter Horiba. XRD measurements were performed using D8 Advance, Bruker-AXS X-ray diffractometer. The powder samples were dispersed in toluene and dropcasted onto a glass slide for the XRD measurement ( $\mathrm{Cu} \mathrm{K} \alpha$, wavelength $\lambda=1.5406 \mathrm{~A}^{\circ}$ ). Microscopic images were captured using SEM (JSM-7001F, JEOL) and TEM (JEOL, JEM-2100). EDS analysis was carried out from TEM measurements. TCA was measured using TGA/ SDTA851e/LF/1600 instrument and the temperature range was from ambient temperature to $1000{ }^{\circ} \mathrm{C}$. The temperature increment rate was $5{ }^{\circ} \mathrm{C} \mathrm{min}{ }^{-1}$. RAMAN spectroscopic measurement for the powder samples was carried out using RAMAN WITEC APYRON instrument, the laser wavelength for all the samples were $785 \mathrm{~nm}$.

XPS measurements were performed using a Kratos AXIS Ultra DLD instrument. The chamber pressure during the measurements was $5 \times 10$ -9 Torr. Wide energy range survey scans were collected at a pass energy of $80 \mathrm{eV}$ in hybrid slot lens mode and a step size of $0.5 \mathrm{eV}$, for $20 \mathrm{~min}$. The charge neutralizer filament was used to prevent the sample charging over the irradiated area. The X-ray source was a monochromated Al $K_{\alpha}$ emission, run at $10 \mathrm{~mA}$ and $12 \mathrm{kV}(120 \mathrm{~W})$. The energy range for each "pass energy" (resolution) was calibrated using the Kratos Cu 2p 3/2, Ag $3 \mathrm{~d} 5 / 2$, and $A u 4 f 7 / 2$ three-point calibration method. The transmission function was calibrated using a clean gold sample method for all lens modes and the Kratos transmission generator software within Vision II. The data were processed with CASAXPS (Version 2.3.17). The highresolution data were charge corrected to the reference $C$ is signal at $285 \mathrm{eV}$.

For low temperature PL and time-resolved PL (TRPL) measurements, the samples were held in the coldfinger of a closed-cycle He cryostat (ARS DE-202), which can be cooled down to $15 \mathrm{~K}$. PL was excited by means of $200 \mathrm{fs}$ pulsed Ti:sapphire passive mode-locked laser (Coherent

Table 1. Details of flow-rates and obtained materials.

\begin{tabular}{lccccc}
\hline Entry & Precursor flow-rate $\left[\mathrm{mL} \mathrm{min}^{-1}\right]$ & Antisolvent flow-rate $\left[\mathrm{mL} \mathrm{min}^{-1}\right]$ & Obtained material & Emission under $365 \mathrm{~nm}$ UV lamp & Stability \\
\hline 1 & 0.1 & 1 & $\mathrm{CS}_{4} \mathrm{SnBr}_{6}$ & Green & Few hours \\
2 & 0.2 & 1 & $\mathrm{Cs}_{4} \mathrm{SnBr}_{6}$ & Green & Few hours \\
3 & 0.3 & 1 & $\mathrm{Sn}$ (II)-doped CsBr & Orange & Over 7 months \\
4 & 0.4 & 1 & $\mathrm{Sn}$ (II)-doped CsBr & Orange & Over 7 months \\
5 & 0.5 & 1 & Sn (II)-doped CsBr & Orange & Over 7 months \\
\hline
\end{tabular}


Mira 900D, $76 \mathrm{MHz}$ repetition rate) operating at a wavelength of $810 \mathrm{~nm}$ and doubled to $405 \mathrm{~nm}$ by using a BBO crystal. The PL signal was dispersed by a double $03 \mathrm{~m}$ focal length grating spectrograph (Acton SP-300i from Princeton Instruments) and detected with a cooled Si charge-coupled device camera (Newton EMCCD from ANDOR) for $\mathrm{PL}$ spectral measurements and with a silicon single photon avalanche photodiode (micro photon device) connected to a time-correlated single-photon counting electronic board (TCC900 from Edinburgh Instruments) for TRPL measurements.

\section{Supporting Information}

Supporting Information is available from the Wiley Online Library or from the author.

\section{Acknowledgements}

This work was partially supported by the European Research Council (ERC) via Consolidator Grant (724424-No-LIMIT) and the European Commission via FETOpen Grant (862656-DROP-IT). The authors acknowledge SCIC from Jaume I University (UII) for help with XRD, SEM, TGA, and Raman characterization. J.A.F. would like to thank the University of Nottingham Beacons of Excellence: Propulsion Futures and EPSRC: LiPPS XPS system, and EP/K005138/1 "University of Nottingham Equipment Account" for providing financial support for this work and the nanoscale and microscale research center (University of Nottingham, UK) for access to XPS facilities.

\section{Conflict of Interest}

The authors declare no conflict of interest.

\section{Data Availability Statement}

Research data are not shared.

\section{Keywords}

continuous flow reactor synthesis, $\mathrm{CsBr}$, interstitial doping, long-term ambient stability, photoluminescence quantum yield, self-trapped excitonic emission

Received: May 21, 2021

Revised: July 23, 202

Published online:

[1] J. Jeong, M. Kim, J. Seo, H. Lu, P. Ahlawat, A. Mishra, Y. Yang, M. A. Hope, F. T. Eickemeyer, M. Kim, Y. I. Yoon, I. W. Choi, B. P. Darwich, S. J. Choi, Y. Jo, J. H. Lee, B. Walker, S. M. Zakeeruddin, L. Emsley, U. Rothlisberger, A. Hagfeldt, D. S. Kim, M. Grätzel, J. Y. Kim, Nature 2021, 592, 381.

[2] W. Xu, Q. Hu, S. Bai, C. Bao, Y. Miao, Z. Yuan, T. Borzda, A. J. Barker, E. Tyukalova, Z. Hu, M. Kawecki, H. Wang, Z. Yan, X. Liu, X. Shi, K. Uvdal, M. Fahlman, W. Zhang, M. Duchamp, J.-M. Liu, A. Petrozza, J. Wang, L.-M. Liu, W. Huang, F. Gao, Nat. Photonics 2019, 13, 418

[3] C. Chen, D. Li, Y. Wu, C. Chen, Z.-G. Zhu, W. Y. Shih, W.-H. Shih, Nanotechnology 2020, 31, 225602.
[4] C. Chen, Y. Wu, Z.-G. Zhu, W. Y. Shih, W.-H. Shih, J. Mater. Res. 2021, 36, 1835

[5] J. Lin, Y. Lu, X. Li, F. Huang, C. Yang, M. Liu, N. Jiang, D. Chen, ACS Energy Lett. 2021, 6, 519

[6] S. Yuan, D. Chen, X. Li, J. Zhong, X. Xu, ACS Appl. Mater. Interfaces 2018, 10, 18918.

[7] J. Li, H.-L. Cao, W.-B. Jiao, Q. Wang, M. Wei, I. Cantone, J. Lü, A. Abate, Nat. Commun. 2020, 11, 310.

[8] M. Lyu, J.-H. Yun, P. Chen, M. Hao, L. Wang, Adv. Energy Mater. 2017, 7, 1602512.

[9] S. M. Jain, T. Edvinsson, J. R. Durrant, Commun. Chem. 2019, 2, 91.

[10] P. V. Kamat, J. Bisquert, J. Buriak, ACS Energy Lett. 2017, 2, 904

[11] B. Saparov, F. Hong, J.-P. Sun, H.-S. Duan, W. Meng, S. Cameron, I. G. Hill, Y. Yan, D. B. Mitzi, Chem. Mater. 2015, 27, 5622.

[12] A. H. Slavney, T. Hu, A. M. Lindenberg, H. I. Karunadasa, J. Am. Chem. Soc. 2016, 138, 2138.

[13] T.-B. Song, T. Yokoyama, S. Aramaki, M. G. Kanatzidis, ACS Energy Lett. 2017, 2, 897.

[14] H. Chen, J. M. Pina, F. Yuan, A. Johnston, D. Ma, B. Chen, Z. Li, A. Dumont, X. Li, Y. Liu, S. Hoogland, Z. Zajacz, Z. Lu, E. H. Sargent, J. Phys. Chem. Lett. 2020, 11, 4326.

[15] J. Luo, X. Wang, S. Li, J. Liu, Y. Guo, G. Niu, L. Yao, Y. Fu, L. Gao, Q. Dong, C. Zhao, M. Leng, F. Ma, W. Liang, L. Wang, S. Jin, J. Han, L. Zhang, J. Etheridge, J. Wang, Y. Yan, E. H. Sargent, J. Tang, Nature 2018, 563, 541.

[16] J. Li, J. Duan, X. Yang, Y. Duan, P. Yang, Q. Tang, Nano Energy 2021, 80, 105526

[17] Z. Zhang, R. Zhao, S. Teng, K. Huang, L. Zhang, D. Wang, W. Yang, R. Xie, N. Pradhan, Small 2020, 16, 2004272.

[18] E. López-Fraguas, S. Masi, I. Mora-Seró, ACS Appl. Energy Mater. 2019, 2, 8381

[19] S. Masi, A. F. Gualdrón-Reyes, I. Mora-Seró, ACS Energy Lett. 2020, 5, 1974.

[20] Z. Wang, J. Zhang, W. Guo, W. Xiang, A. Hagfeldt, Matter 2021, 4, 528.

[21] J. Cao, F. Yan, Energy Environ. Sci. 2021, 14, 1286.

[22] X. Li, X. Gao, X. Zhang, X. Shen, M. Lu, J. Wu, Z. Shi, V. L. Colvin, J. Hu, X. Bai, W. W. Yu, Y. Zhang, Adv. Sci. 2021, 8, 2003334.

[23] M. Li, Z. Xia, Chem. Soc. Rev. 2021, 50, 2626.

[24] K. M. McCall, V. Morad, B. M. Benin, M. V. Kovalenko, ACS Mater. Lett. 2020, 2, 1218

[25] A. E. Maughan, A. M. Ganose, D. O. Scanlon, J. R. Neilson, Chem. Mater. 2019, 31, 1184.

[26] T. C. Jellicoe, J. M. Richter, H. F. J. Glass, M. Tabachnyk, R. Brady, S. E. Dutton, A. Rao, R. H. Friend, D. Credgington, N. C. Greenham, M. L. Böhm, J. Am. Chem. Soc. 2016, 138, 2941.

[27] B. M. Benin, D. N. Dirin, V. Morad, M. Wörle, S. Yakunin, G. Rainò, O. Nazarenko, M. Fischer, I. Infante, M. V. Kovalenko, Angew. Chem., Int. Ed. 2018, 57, 11329.

[28] R. Chiara, Y. O. Ciftci, V. I. E. Queloz, M. K. Nazeeruddin, G. Grancini, L. Malavasi, J. Phys. Chem. Lett. 2020, 11, 618.

[29] X. Zhang, H. Wang, S. Wang, Y. Hu, X. Liu, Z. Shi, V. L. Colvin, S. Wang, W. W. Yu, Y. Zhang, Inorg. Chem. 2020, 59, 533.

[30] L. Tan, W. Wang, Q. Li, Z. Luo, C. Zou, M. Tang, L. Zhang, J. He, Z. Quan, Chem. Commun. 2020, 56, 387.

[31] V. Santhana, D. C. Greenidge, D. Thangaraju, R. Marnadu, T. Alshahrani, M. Shkir, Mater. Lett. 2020, 280, 128562.

[32] S. Li, J. Luo, J. Liu, J. Tang, J. Phys. Chem. Lett. 2019, 10 1999.

[33] A. Veronese, M. Patrini, D. Bajoni, C. Ciarrocchi, P. Quadrelli, L. Malavasi, Front. Chem. 2020, 8, 35.

[34] I. Lignos, S. Stavrakis, G. Nedelcu, L. Protesescu, A. J. deMello, M. V. Kovalenko, Nano Lett. 2016, 16, 1869. 
[35] R. M. Maceiczyk, K. Dümbgen, I. Lignos, L. Protesescu, M. V. Kovalenko, A. J. deMello, Chem. Mater. 2017, 29, 8433.

[36] I. Lignos, L. Protesescu, D. B. Emiroglu, R. Maceiczyk, S. Schneider, M. V. Kovalenko, A. J. deMello, Nano Lett. 2018, 18, 1246.

[37] X. Liang, R. W. Baker, K. Wu, W. Deng, D. Ferdani, P. S. Kubiak, F. Marken, L. Torrente-Murciano, P. J. Cameron, React. Chem. Eng. 2018, 3, 640 .

[38] O. Okafor, K. Robertson, R. Goodridge, V. Sans, React. Chem. Eng. 2019, 4, 1682

[39] O. Okafor, A. Weilhard, J. A. Fernandes, E. Karjalainen, R. Goodridge, V. Sans, React. Chem. Eng. 2017, 2, 129.

[40] V. Sans, S. Glatzel, F. J. Douglas, D. A. Maclaren, A. Lapkin, L. Cronin, Chem. Sci. 2014, 5, 1153.

[41] S. Yakunin, B. M. Benin, Y. Shynkarenko, O. Nazarenko, M. I. Bodnarchuk, D. N. Dirin, C. Hofer, S. Cattaneo, M. V. Kovalenko, Nat. Mater. 2019, 18, 846.
[42] A. Kaltzoglou, M. Antoniadou, A. G. Kontos, C. C. Stoumpos, D. Perganti, E. Siranidi, V. Raptis, K. Trohidou, V. Psycharis, M. G. Kanatzidis, P. Falaras, J. Phys. Chem. C 2016, 120, 11777.

[43] T.-B. Song, T. Yokoyama, C. C. Stoumpos, J. Logsdon, D. H. Cao, M. R. Wasielewski, S. Aramaki, M. G. Kanatzidis, J. Am. Chem. Soc. 2017, 139, 836.

[44] P. Giannozzi, O. Andreussi, T. Brumme, O. Bunau, M. Buongiorno Nardelli, M. Calandra, R. Car, C. Cavazzoni, D. Ceresoli, M. Cococcioni, N. Colonna, I. Carnimeo, A. Dal Corso, S. de Gironcoli, P. Delugas, R. A. DiStasio, A. Ferretti, A. Floris, G. Fratesi, G. Fugallo, R. Gebauer, U. Gerstmann, F. Giustino, T. Gorni, J. Jia, M. Kawamura, H. Y. Ko, A. Kokalj, E. Küçükbenli, M. Lazzeri, M. Marsili, N. Marzari, F. Mauri, N. L. Nguyen, H. V. Nguyen, A. Otero-de-la-Roza, L. Paulatto, S. Poncé, D. Rocca, R. Sabatini, B. Santra, M. Schlipf, A. P. Seitsonen, A. Smogunov, I. Timrov, T. Thonhauser, P. Umari, N. Vast, X. Wu, S. Baroni, J. Phys.: Condens. Matter 2017, 29, 465901.

[45] K. Teegarden, G. Baldini, Phys. Rev. 1967, 155, 896. 